\title{
Surface Brightness Measurements of Extended Galactic Nebulae
}

\author{
Ronald Stark ${ }^{1}$ and Klaus Reif ${ }^{2}$ \\ ${ }^{1}$ Max-Planck-Institut für Radioastronomie, Auf dem Hügel 69, D-53121 Bonn, Germany \\ stark@mpifr-bonn.mpg.de \\ ${ }^{2}$ Sternwarte, Universität Bonn, Auf dem Hügel 71, D-53121 Bonn, Germany
}

Received 1997 August 1, accepted $199^{7}$ December 9

\begin{abstract}
We discuss the importance of digitised sky-limited Schmidt B- and R-band, and $\mathrm{H} \alpha$ images of extended translucent $\left(A_{\mathrm{B}} \simeq 1-5 \mathrm{mag}\right)$ and dark $\left(A_{\mathrm{B}}>5\right.$ mag) clouds. In translucent clouds (e.g. high-latitude cirrus) photons can penetrate through the whole cloud and control the physical and chemical processes. Comparison of the B images with far-infrared/submillimetre CO, CI, and Ciı emission provides important information on the non-homogeneous cloud structure, carbon chemistry and energy balance. In dark clouds the interior is completely shielded from the interstellar radiation field. L1204 is a dark cloud with at its edge the well-known Hir rim S140. The R-band image shows S140 as well as the diffuse extended Hir region around the exciting B-type star. However, the sensitivity for $\mathrm{H} \alpha$ in the R-band is rather low and there is contamination with diffusely scattered red light from dust. Deep $\mathrm{H} \alpha$ and R-band imaging of an $\sim 20^{\prime} \times 20^{\prime}$ field has recently been done with the $1 \mathrm{~m}$ Hoher List telescope of Bonn University. A wealth of small-scale structures can been seen on continuum-subtracted images which provide new insight on the geometry of this cloud.
\end{abstract}

Keywords: ISM: clouds, dust, individual (cirrus, L1204/S140) — reflection nebulae: structure

\section{A Case for Schmidt Photography anno 1997}

These days the photographic plate has become a rarely used detector in astronomy. Imaging is now commonly done with CCDs which have a much higher quantum efficiency and a linear response. In addition, the general tendency is to make larger and larger telescopes with a decreasing detector field of view, which is often less than a few arcmin. This makes them unsuitable for studying degreesized astronomical objects. In contrast, IIIa-type and Technical Pan (Tech Pan) detectors (Schmidt plates/films) have a high-contrast emulsion and a large photon detection area (e.g. UKST $\sim 40$ square degrees). They are therefore excellently suited to the study of extended objects with low surface brightness, e.g. low surface brightness galaxies (Phillips \& Parker 1993; Schwartzenberg, Phillips \& Parker 1995, 1996) and galactic clouds (Stark 1995). A disadvantage of photographic emulsion is its non-linearity. However, for low surface brightness objects a small increase in photographic density is linearly correlated with a small step in surface brightness. This means that for high-quality uniform plates the relative surface brightness can be derived directly, and calibrated if the sky brightness is measured during the exposure. In this way faint objects with $\mu_{\mathrm{B}_{\mathrm{J}}} \sim 25-27 \mathrm{mag} / \operatorname{arcsec}^{2}$ can readily be studied (de Vries \& Le Poole 1985;
Stark 1995) with IIIa-J plates. The sensitivity of the IIIa-F emulsions used for R-band imaging is about the same as that of IIIa-J, but the night sky is about a magnitude brighter in the R-band, so the detection of faint objects is correspondingly poorer. With the advent of Tech Pan films, which have finer grains than IIIa emulsions and a much higher detection quantum efficiency, it is possible to study faint structures in $\mathrm{R}$ and $\mathrm{H} \alpha$ down to a brightness limit which is comparable to those for IIIa-J emulsions (Phillips \& Parker 1993). The best way for a detailed quantitative analysis is to digitise Schmidt films/plates at a resolution which properly samples the point-spread function of the system (seeing, telescope and emulsion), i.e. with $10 \mu \mathrm{m}$ pixels $\equiv 0.67$ arcsec (see MacGillivray 1998, this issue p. 42).

In this paper we will discuss surface brightness measurements of galactic clouds in comparison with far-infrared/submillimetre CO, CI, and CII emission. We distinguish between diffuse $\left(A_{\mathrm{B}}<1\right.$ mag), translucent $\left(A_{\mathrm{B}} \simeq 1-5 \mathrm{mag}\right)$ and dark $\left(A_{\mathrm{B}}>5\right.$ mag) clouds.

\section{Every Cloud has a Translucent Region}

The physical and chemical processes in translucent clouds are controlled by the interstellar radiation 
field (ISRF) which can penetrate through the whole cloud, although at a reduced rate into the deepest parts. A well known example of these clouds are the ubiquitously present high-latitude IRAS cirrus. It is noteworthy that these cirrus had already been discovered before IRAS on high-quality IIIa-J plates taken at UKST in the course of the deep ESO/SERC southern sky survey (e.g. King, Taylor \& Tritton 1979).

Optical surface brightness images provide important information for the investigation of the internal structure of translucent clouds or cloud regions. It is important to realise that every cloud has a translucent layer: the edges of dark clouds, giant molecular clouds, and, in the ultimate sense, the edge of every clump is a translucent region. An important chemical aspect of such regions is that they trace the regime where the carbon transformation $\mathrm{C}$ $\rightarrow \mathrm{C}^{+} \rightarrow \mathrm{CO}$ takes place (e.g. van Dishoeck \& Black 1988; Stark \& van Dishoeck 1994). This transition is very sensitive to the physical parameters, and small variations in e.g. temperature, total hydrogen density and column density can cause large variations in the observed $\mathrm{CO}$ emission. In a detailed study of a series of isolated high-latitude cirrus clouds it was found that a smooth blue surface brightness distribution corresponds often to a filametary ${ }^{12} \mathrm{CO}$ emission without significant ${ }^{13} \mathrm{CO}$ emission (Stark 1995). On the other hand, when brightness condensations are visible on the B-image they coincide often with clumps in the ${ }^{12} \mathrm{CO}$ emission where also ${ }^{13} \mathrm{CO}$ emission becomes significant. The optical surface brightness images are therefore an important tool for distinguishing between volume density and column density variations. There is a very good correspondence between the optical and FIR surface brightness (Paley et al. 1991; Stark 1995). The optical images reveal the diffusely backscattered radiation from stars in the Galactic Plane (Sandage 1976), while the absorbed radiation is re-radiated at FIR wavelengths. Comparison provides direct information on the energy balance.

Optical surface brightness images will be indispensable in identifying spurious effects in future FIR-CBR surveys. First results of a project to study the carbon content in galactic cirrus clouds through comparison of the lowest $\mathrm{CO}$ rotational transitions and observations of the fine-structure line of Ci at 492 GHz (Stark \& van Dishoeck 1994; Ingalls et al. 1994, $1997)$ indicate that most of the carbon is not yet in the form of $\mathrm{CO}$, i.e. $N(\mathrm{C}) / N(\mathrm{CO})>1$. Moreover, recent observations of the CII $158 \mu \mathrm{m}$ line with the Infrared Space Observatory (ISO) shows this line to have a strength comparable to CI which indicates a low incident radiation field ${ }^{1}, I_{\mathrm{UV}}<0.5$ (Stark et al. in preparation). The clouds are therefore cold

\footnotetext{
${ }^{1}$ Throughout this paper the ultraviolet intensity of the radiation field is expressed in units of the general ISRF as given by Draine (1978)
}

$\left(T_{\text {dust }} \sim 15 \mathrm{~K}\right)$ and radiate much of their energy at wavelengths greater than $200 \mu \mathrm{m}$. Understanding small-scale surface brightness variations of foreground cirrus at optical wavelenghths will therefore be of vital importance for projects searching for variations of the FIR CBR with submillimetre bolometer arrays.

\section{Structure and Geometry of L1204/S140}

L1204/S140 is a classical, well studied edge-on photon-dominated region (PDR). It consists of the dark cloud L1204, D $\simeq 910 \mathrm{pc}$ (Crampton \& Fisher 1974), which is bordered in the south-west by an HII region. This HII region is commonly thought to be excited by the B0 V star HD 211880 located well outside the cloud. The interface between the cloud and HII region is visible as a bright rim called S140 (Sharpless 1959), located at $7^{\prime} \mathrm{NE}$ of the exciting star. At $1^{\prime}$ north-east of this interface lies a dense core with $A_{\mathrm{V}} \simeq 30 \mathrm{mag}$, which is associated with a cluster of infrared objects (Beichman, Becklin \& Wynn-Williams 1979). These have been identified as embedded early B-type stars (Evans et al. 1989) and are commonly denoted as S140IRS. One of them, IRS1, is associated with a bipolar molecular outflow (Bally \& Lada 1983) along a NW-SE axis.

The S140IRS region has been intensively studied in molecular line emission at high angular resolution. Only recently studies of the large-scale structure of the L1204 cloud have been performed in $\mathrm{C}^{+}$ (Emery et al. 1996), $\mathrm{C}^{0}$ and ${ }^{13} \mathrm{CO}$ (Plume, Jaffe \& Keene 1994) emission and provide important information on the translucent carbon transition region. These studies reveal a large atomic carbon $\left(\mathrm{C}^{0}, \mathrm{C}^{+}\right)$abundance over the whole cloud even at large distances from the interface region. This can be understood from a non-homogeneous cloud structure, where the UV photons can penetrate deep into the cloud, and has been modelled successfully by Spaans \& van Dishoeck (1996), who also investigated geometry effects, e.g. if the illuminating star and L1204 do not lie in the same plane on the sky. Direct information on the translucent cloud structure and geometry are lacking, but can be obtained from deep optical images (see Section 2). We therefore started a program to get deep R, I, and $\mathrm{H} \alpha$ CCD images of the L1204/S140 complex using the 1.06 m Cassegrain telescope of Bonn University at Hoher List Observatory. In combination with a focal reducer $(\mathrm{f} / 3 \cdot 5)$ a field size of $28^{\prime}$ (circular) is obtained with a pixel resolution of $0 \cdot 8^{\prime \prime}$ on a $2 \mathrm{k} \times 2 \mathrm{k}$ CCD. Below we present first results from deep $\mathrm{H} \alpha$ imaging.

Figure 1 shows part of a digitised POSS II R-band film (smoothed to $4^{\prime \prime}$ resolution) centred on S140IRS with superposed contours of the integrated ${ }^{13} \mathrm{CO}(2-1)$ emission ( $3^{\prime}$ resolution; Plume et al. 1994). Note that the ${ }^{13} \mathrm{CO}$ emission appears to extend into the His region. This is not real; due to the low resolution of ${ }^{13} \mathrm{CO}$ the sharp edge is 


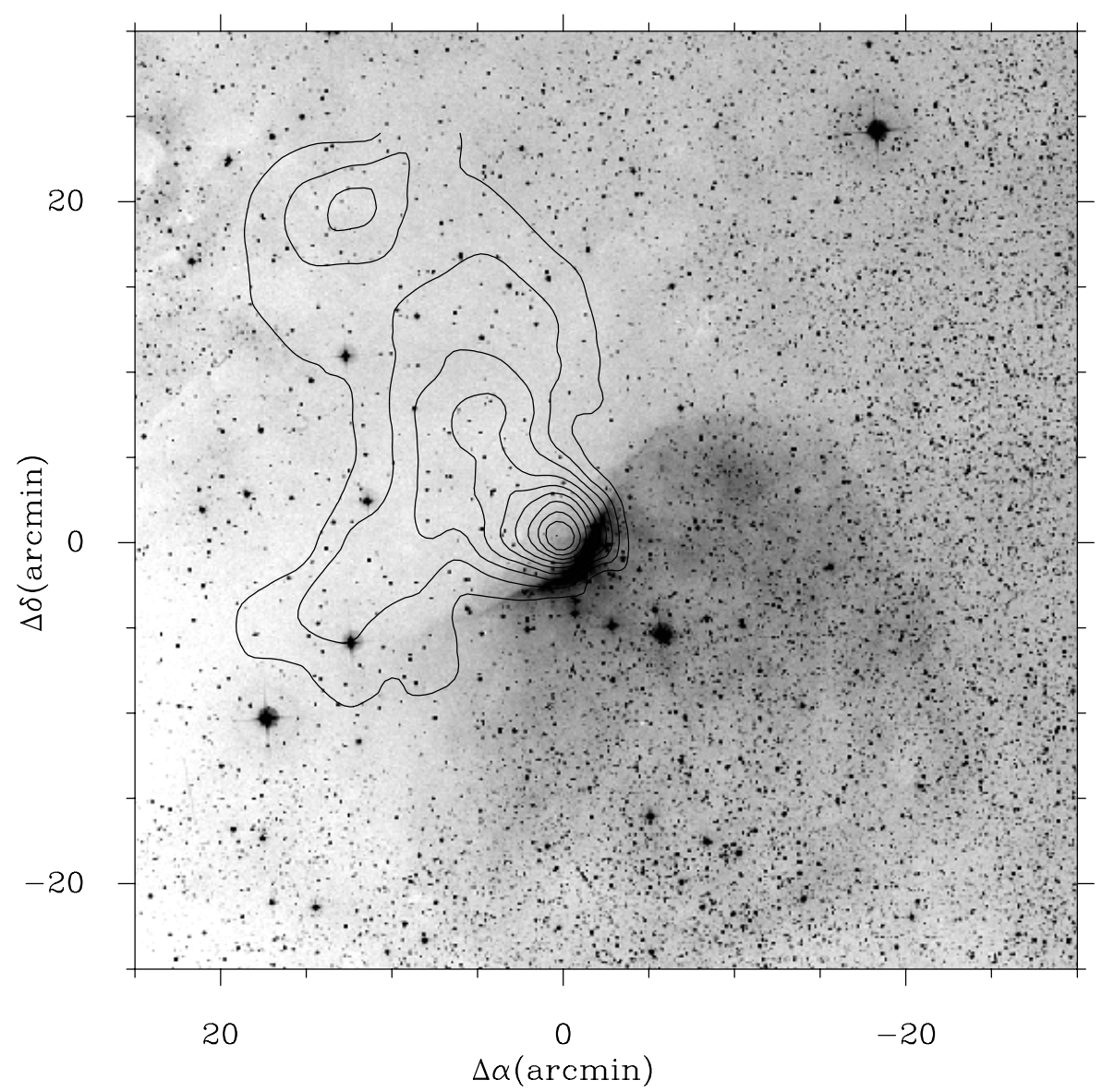

Figure 1-POSS II R-band image of L1204/S140 $\left(55^{\prime} \times 55^{\prime}\right.$, centred on S140IRS1) with superposed contours of the integrated ${ }^{13} \mathrm{CO}(2-1)$ emission (HPBW $=3^{\prime}$; Plume et al. 1994). Darker grey scales correspond to higher brightnesses.

unresolved and therefore appears displaced. The R-band image clearly reveals the dark cloud L1204, the bright rim S140 at $\left(-1^{\prime},-1^{\prime}\right)$, as well as the diffuse extended Hir region around the exciting Btype star at $\left(-6^{\prime},-5 \cdot 5^{\prime}\right)$. However, the sensitivity for the $\mathrm{H} \alpha$ line in the R-band is rather low and there is contamination with diffusely scattered red light from dust. Figure 2 shows the continuum subtracted $\mathrm{H} \alpha$ CCD image. A wealth of small-scale structures is visible on the $\mathrm{H} \alpha$ image. Brightness variations in the diffuse $\mathrm{H} \alpha$ emission are real and caused by extinction of dust within the HiI region. The edge of the dark cloud is well delineated; note also the eruption-like structures in S140 which are typical of photoevaporation. It is also clearly visible that the Hir emission extends over the face of the cloud. Figure 3 shows a NE-SW cut crossing S140. The $\mathrm{H} \alpha$ intensity drops steeply at the interface, but remains significant even at the position of S140IRS. Thus the illumination is somewhat face-on.

The CII emission in the S140 complex has been measured with ISO-LWS by Emery et al. (1996). They found a strong increase in CII intensity at the interface (see their Figure 3), and the emission remains strong at large distances into the cloud. The derived UV (912-2000 A) intensity at the interface is about $I_{\mathrm{UV}} \simeq 100$. This is well matched by the flux of a B0V star at the projected distance of $2 \mathrm{pc}\left(7^{\prime}\right)$. Further into the L1204 cloud the CII emission requires a constant $I_{\mathrm{UV}} \simeq 15$, which would be possible if the star is located $\sim 25^{\circ}$ out of the plane of the cloud. In addition, for a clumpy cloud structure, there may be a contribution of UV flux from the embedded sources. Smirnov, Sorochenko \& Walmsley (1995) have measured the $\mathrm{H} 166 \alpha$ recombination line and derived $\left\langle n_{e}\right\rangle \sim 10$ $\mathrm{cm}^{-3}$. A crude estimate of the Strömgren radius from the $\mathrm{H} \alpha$ image gives $R_{S} \sim 5 \mathrm{pc}$ (for a $\mathrm{B} 0 \mathrm{~V}$ photon flux) which yields an average electron density consistent with theirs. It is interesting that $\mathrm{H} 166 \alpha$ emission was found even in the direction of L1204; this could mean that the HiI region may extend behind the cloud. A detailed analysis of the deep optical images will be presented elsewhere (Stark, Reif \& Bagschik, in preparation).

\section{AAO/UKST H $\alpha$ Galactic Plane Survey}

Deep $\mathrm{H} \alpha$ Schmidt images will provide a wealth of new information on large- and small-scale lowbrightness features. Two issues are of the utmost importance in revealing faint objects with a brightness below that of the night sky: high-quality plates 


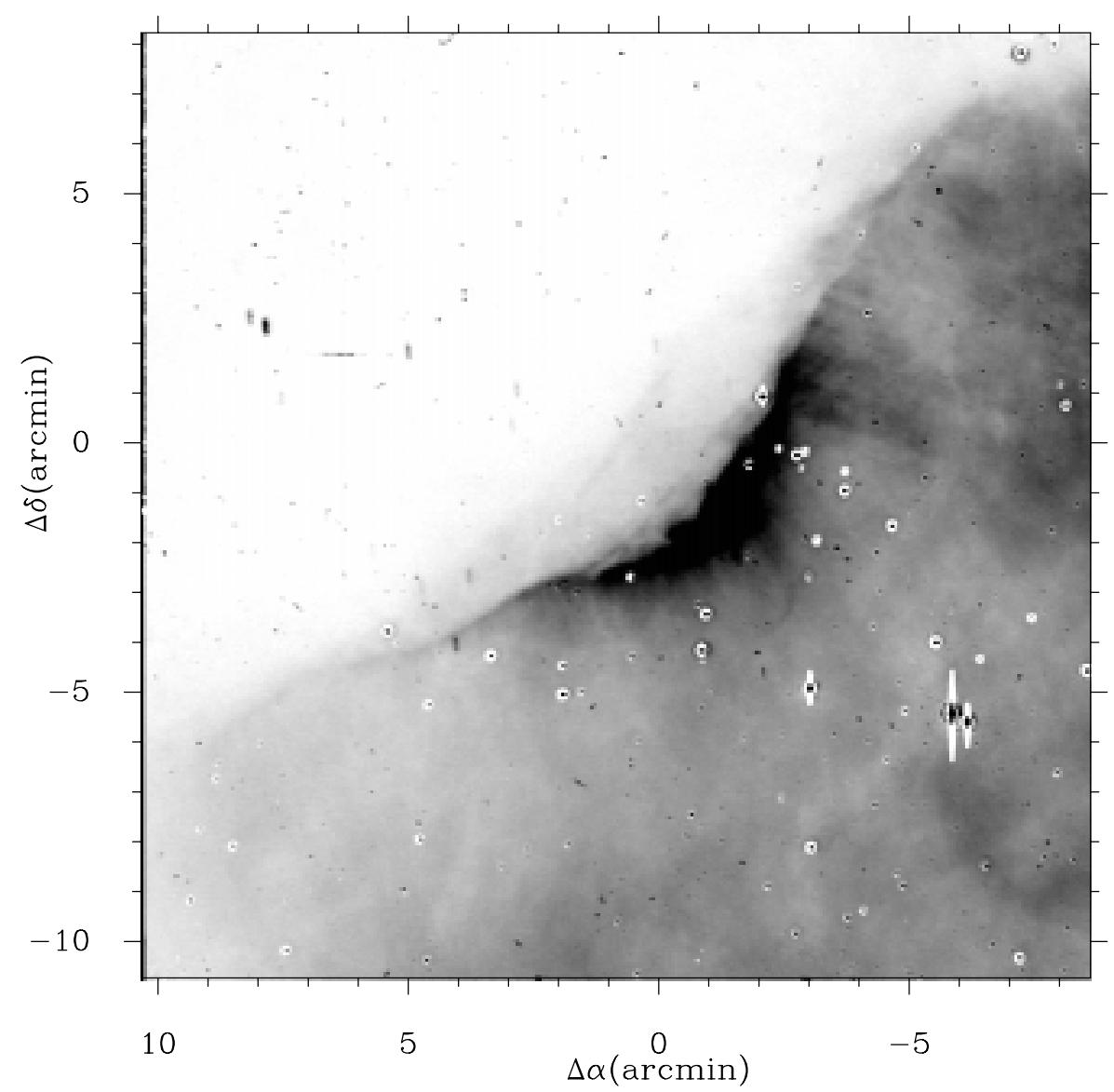

Figure 2-Deep continuum-subtracted H $\boldsymbol{\alpha}$ image of L1204/S140 over an area $19^{\prime} \times 19^{\prime}$ around S140IRS1, taken at the Hoher List Observatory.

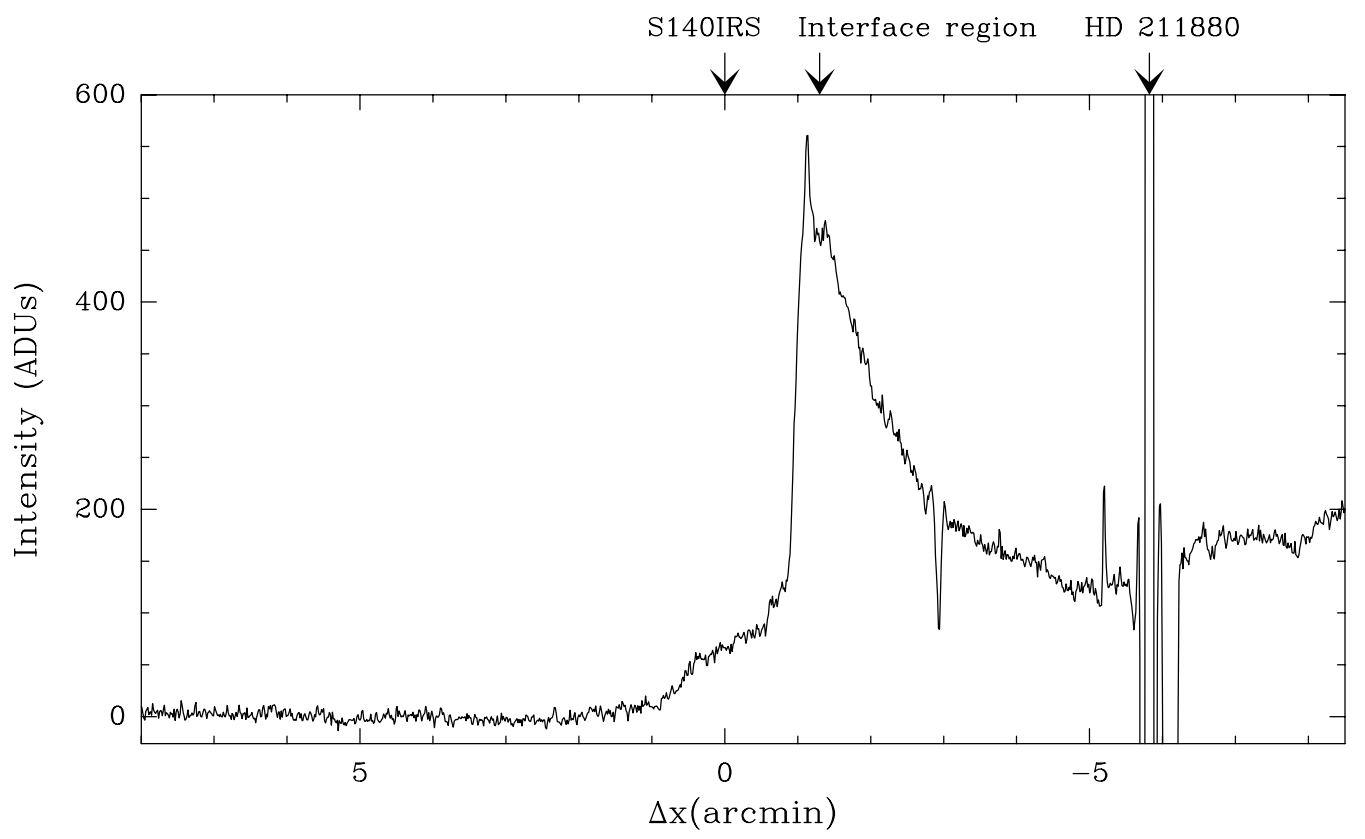

Figure 3-H $\boldsymbol{\alpha}$ intensity along a NE-SW cut through S140IRS and HD 211880.

with a uniform plate background, and monitoring of the sky brightness with a dedicated photometer. These two conditions have become standard only at the AAO/UK Schmidt Telescope. In addition, in this team there has been a continuous effort over last two decades to push Schmidt photography to new limits. The AAO/UK Schmidt Telescope Unit is therefore excellently suited to conduct such a survey. 
A few additional points may be of interest for the $\mathrm{H} \alpha$ Galactic plane survey: (1) Exposures in the $\mathrm{H} \alpha$-continuum filter will be important to separate the dust scattered light component and to reveal true faint $\mathrm{H} \alpha$ features. (2) Excursion to high Galactic latitude fields will be interesting, not only for studying high-latitude clouds (see also Larson 1998, this issue p. 155), but also for deep extragalactic studies, since there will be less confusion with unrelated foreground and background $\mathrm{H} \alpha$ emission. Last but not least, photography remains an art. Besides digitisation of the full plates with SuperCOSMOS (see MacGillivray 1998, this issue p. 42), there will remain an important role for photographic amplification techniques (see Malin 1998, this issue p. 38), in particular since it is a powerful and fast method for revealing new faint objects and making breathtaking astronomical images.

\section{Acknowledgments}

RS kindly thanks the $\mathrm{H} \alpha$ workshop organising committee for travel support. The POSS II Rband film copy was digitised at the Astronomical Institute Münster (AIM). We thank Albert Bruch and Thomas Schimpke of AIM for their support. The $\mathrm{H} \alpha$ image has been taken by Klaus Bagschik from Bonn University.
Bally, J., \& Lada, C. 1983, ApJ, 265, 824

Beichman, C. A., Becklin, E. E., \& Wynn-Williams, C. G. 1979, ApJ, 232, L47

Crampton, D., \& Fisher, W. A. 1974, Publ. Dom. Ap. Obs. 14 , No. 12

de Vries, E. F., \& Black, J. H. 1988, ApJ, 334, 771

Draine, B. T. 1978, ApJS, 36, 595

Emery, R., Aanestad, P., Minchin, N., Unger, S. J., Baluteau, J.-P., et al. 1996, A\&A, 315, L285

Evans, N. J. II, Mundy, L. G., Kutner, M. L., \& DePoy, D. L. 1989, ApJ, 346, 212

Ingalls, J. G., Bania, T. M., \& Jackson, J. M. 1994, ApJL, 431, 139

Ingalls, J. G., et al. 1997, ApJ, 479, 296

King, D. J., Taylor, K. N. R., \& Tritton, K. P. 1979, MNRAS, 188, 719

Larson, K. 1998, PASA, 15, 155

MacGillivray, H. 1998, PASA, 15, 42

Malin, D. 1998, PASA, 15, 38

Paley, E. S., Low, F. J., McGraw, J. T., Cutri, R. M., \& Rix, H.-W. 1991, ApJ, 376, 335

Phillipps, S., \& Parker Q. A. 1993, MNRAS, 265, 385

Plume, R., Jaffe, D. T., \& Keene, J. 1994, ApJL, 425, 49

Sandage, A. 1976, AJ, 81, 954

Schwartzenberg, J. M., Phillipps, S., \& Parker, Q. A. 1995, A\&A, 293, 332

Schwartzenberg, J. M., Phillipps, S., \& Parker, Q. A. 1996, A\&AS, 117, 179

Sharpless, S. 1959, ApJS, 4, 257

Smirnov, G. T., Sorochenko, R. L., \& Walmsley, C. M. 1995, A\&A, 300, 923

Spaans, M., \& van Dishoeck, E. F. 1997, A\&A, 373, 953

Stark, R. 1995, A\&A, 301, 873

Stark, R., \& van Dishoeck, E. F. 1994, A\&A, 386, L43

van Dishoeck, C. P., \& Le Poole, R. S. 1985, A\&A, 145, L7 